\title{
Solar Gamma-Ray Line Spectroscopy - Physics of a Flaring Star
}

\author{
Gerald H. Share and Ronald J. Murphy \\ E.O. Hulburt Center for Space Research, Naval Research Laboratory, \\ 4555 Overlook Ave., S.W., Washington, DC 20375, USA
}

\begin{abstract}
We discuss how gamma-ray spectroscopy provides information about the acceleration and transport of electrons and ions in solar flares, and their interaction with the solar atmosphere. Temporal studies illuminate differences in the acceleration and transport of electrons and ions. Nuclear line studies reveal the elemental abundance, density, and temperature of the ambient solar atmosphere; and the spectrum, composition, and directionality of the accelerated ions.
\end{abstract}

\section{Introduction}

This conference focuses on understanding the physics of the Sun and its cool-star counterparts. In this paper we discuss the physics of flares as revealed in the gamma-ray energy band. Chupp et al. (1973) first observed gamma-ray lines from intense solar flares on 1972 August 4 and 7 using the $7^{\text {th }}$ Orbiting Solar Observatory. Since then gamma-ray lines have been observed in $\sim 100$ flares. It is unlikely that we will detect a gamma-ray line flare from another star in the foreseeable future. The most intense solar flare producing gamma-rays would only be detectable within $44 \mathrm{AU}$ with current instruments. Instruments $10^{3} \times$ more sensitive might barely detect a flare on $\alpha$ Cen (4.4 light years distant) if it were $10^{4} \times$ the intensity of the largest solar flare. We will therefore concentrate our discussion on the solar observations made over the past 23 years, with emphasis on the observation of the 2003 July 23 flare by the Reuven Ramaty High Energy Solar Spectroscopic Imager (RHESSI).

\section{Gamma-Ray Production During Solar Flares}

The Sun is a prolific source of high-energy electrons, ions, and neutrons during flares. These events can produce particles at the flare site with energies extending above a $\mathrm{GeV}$, as revealed by high-energy gamma-ray observations made by the EGRET experiment on The Compton Gamma Ray Observatory (Kanbach et al. 1993). The Sun's magnetic field is the primary energy source for particle acceleration. How this conversion of magnetic energy to kinetic energy takes place is still not known, however, reconnection is a likely mechanism (Miller et al. 1997; Aschwanden 2002).

We show a simplified illustration of the acceleration, transport, and interaction of flare-accelerated particles in Figure 1. The illustration basically reflects 


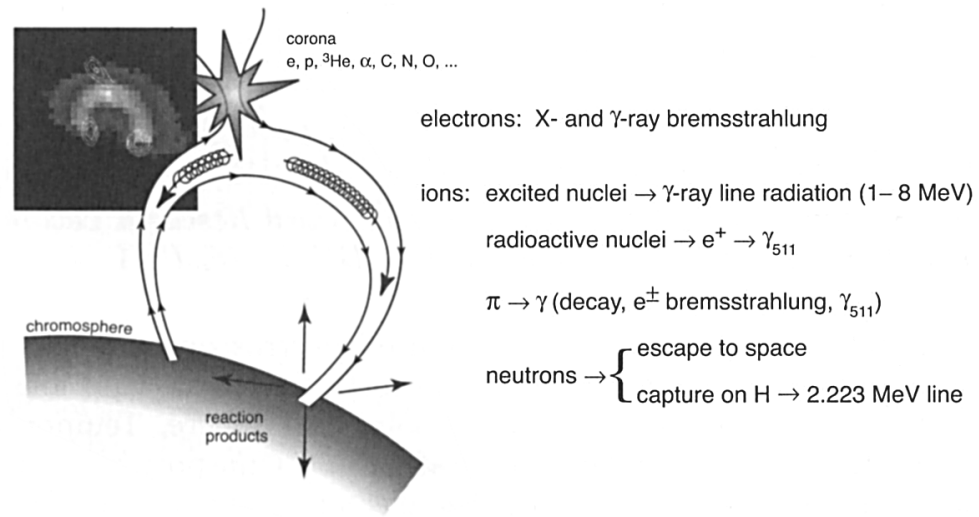

Figure 1. Schematic showing particle acceleration, transport, and interaction products during solar flares

the image in the upper left corner that was taken during a limb flare by the Yohkoh satellite (Masuda et al. 1994). The contours in the image show hard Xray sources and the gray scale shows the image in soft $\mathrm{X}$ rays, which reveals the full magnetic loop filled with hot plasma. In contrast the hard X rays reveal the footpoints of the loops where the accelerated particles interact. A hard X-ray source also appears above the soft X-ray loop. This region may represent the location where the energy for the flare originates. The hard X-ray intensities from the foot points are often different from one another and have an inverse relationship with microwave emissions. This suggests that the electrons have a higher probability of impacting the solar atmosphere where the magnetic field is weaker. The illustration assumes that acceleration occurs at or above the loop top source. Aschwanden et al. (1996) has used timing observations of the hard X-rays from flares to infer that electron acceleration occurs above the looptop hard X-ray source. In contrast, stochastic acceleration of ions by cascading waves requires acceleration lengths $>10^{8} \mathrm{~cm}$, which could encompass a large section of the coronal loop Miller (2000).

Electrons interact to produce bremsstrahlung that reflects their angular distribution. Vestrand et al. (1987) have made a statistical study of the bremsstrahlung continuum in Solar Maximum Mission ( $S M M$ ) flares and conclude that the interacting electron distribution is highly directional. The data are consistent with a downwardly directed Gaussian or a pancake distribution parallel to the solar surface and can be explained by detailed models of electron acceleration and transport (Miller \& Ramaty 1989; McTiernan \& Petrosian 1990). McTiernan \& Petrosian (1990) also calculated the expected bremsstrahlung spectrum and show that it should harden in the few hundred $\mathrm{keV}$ region. The magnitude of this hardening is not sufficient to explain the spectral changes observed by the HXRBS and GRS experiments on $S M M$ shown in Figure 2 and by RHESSI. 


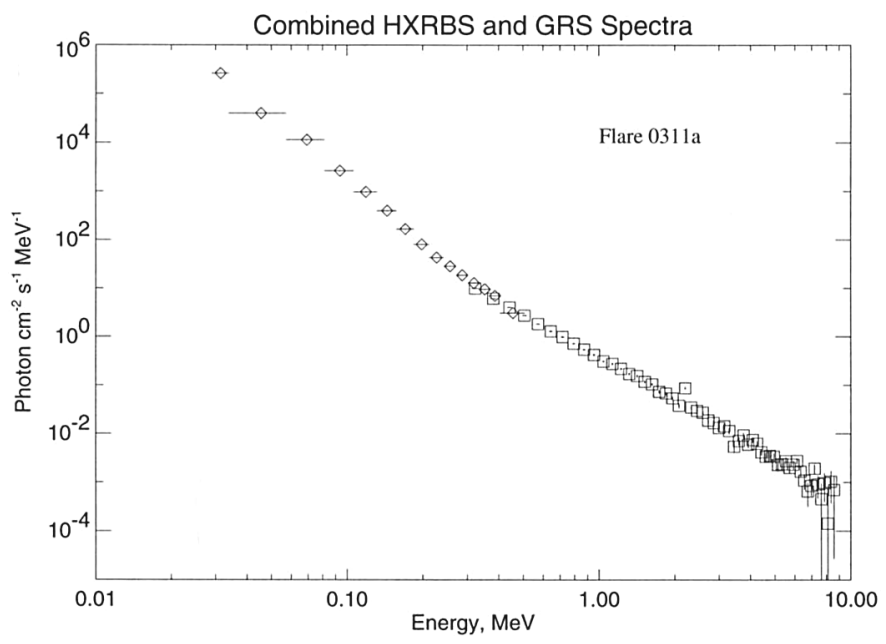

Figure 2. Observed gamma-ray spectrum from a flare on 1980 November 6 exhibiting evidence for spectral hardening with increasing energy.

The ions are transported along the loop where they can encounter MHD waves and undergo pitch angle scattering (PAS) (Hua, Ramaty, and Lingenfelter 1989; Hua et al. 2002). If there is no PAS, then particles with large initial pitch angles will mirror and likely interact there because of the higher density; a smaller number of the particles with small pitch angles will enter the loss cone and interact in a downward direction, deeper in the atmosphere. Without PAS, most particles would be moving parallel to the solar surface when they interact (assuming magnetic loops are perpendicular to the solar surface), similar to a fan beam. PAS causes more particles to enter the loss cone and produces a more downward directed interacting particle distribution.

Ions interact with the ambient material and produce elevated nuclear states that de-excite in the emission of gamma rays characteristic of the element (Ramaty, Kozlovsky, and Lingenfelter 1979; Kozlovsky, Murphy, and Ramaty 2002). The width of the line is broadened due to Doppler recoil and is dependent on the mass of the incident particle, its spectrum, and its angular distribution. Protons and $\alpha$-particles interact with nuclei such as $\mathrm{C}, \mathrm{O}, \mathrm{Ne}, \mathrm{Mg}, \mathrm{Si}$, and $\mathrm{Fe}$ to produce relatively narrow lines. The widths of the $\mathrm{C}$ line excited by protons and $\alpha$-particles are $\sim 75$ and $110 \mathrm{keV}$ (FWHM), respectively. Accelerated heavy ions such as $\mathrm{C}$ interact with ambient $\mathrm{H}$ to produce much broader lines that have widths that are about $30 \%$ of the line energy. Relative line fluxes observed from flares (Share \& Murphy 1995) have been used by Ramaty et al. (1996) to infer that there is a strong FIP effect where the particles interact, suggesting that the ambient abundance has a coronal character. The ion interactions produce a large number of neutrons some of which escape from the Sun and decay in flight or are detected at Earth (Chupp et al. 1987). Neutrons directed toward the Sun slow down and can be captured by ${ }^{1} \mathrm{H}$ in the photosphere to form ${ }^{2} \mathrm{H}$ with the release of energy in the form of a $2.223 \mathrm{MeV}$ gamma ray. Several radioactive nuclei 


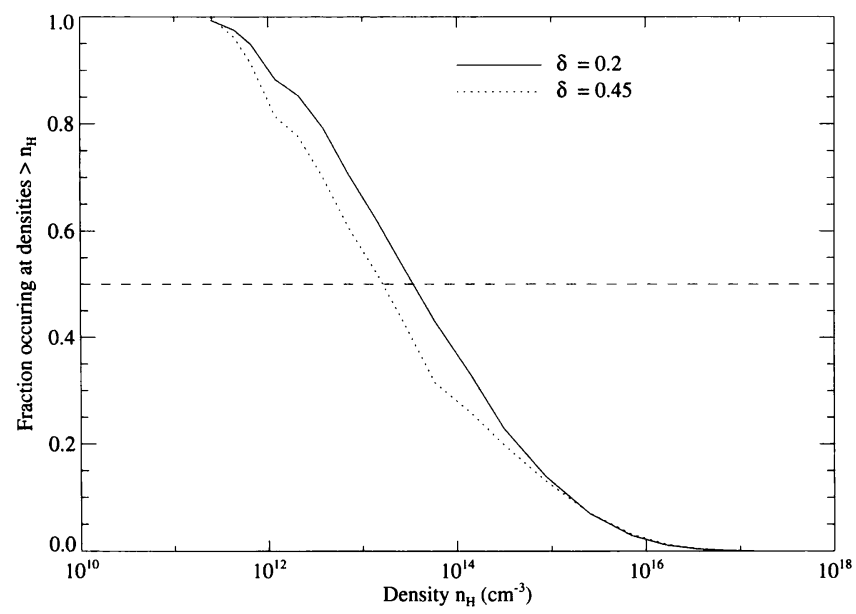

Figure 3. Density at which accelerated protons and $\alpha$ - particles $(\alpha / p$ $=0.5$ ) interact; plotted is the integral number interacting at densities above the value on the abscissa.

that emit positrons are also produced; these positrons slow down and annihilate in flight with the emission of two $511 \mathrm{keV}$ photons or form positronium with the emission of either a three gamma continuum (each photon $<511 \mathrm{keV}$ ) or two $511 \mathrm{keV}$ photons. Higher energy protons and $\alpha$-particles produce charged and neutral pions that decay to produce high-energy electrons/positrons and photons, respectively; these were detected in the 1991 June 11 flare by EGRET (Kanbach et al. 1993).

The depth in the chromosphere where particles interact is dependent on their spectrum and angular distribution, as well as on the convergence of the magnetic field and the atmospheric model. In Figure 3, we plot the fraction of particles producing narrow de-excitation lines at densities greater than listed for two different convergence parameters for: a power-law spectral index of 4.2, a sunspot active region model (Avrett 1981), an assumed $\alpha / \mathrm{p}$ ratio of 0.5 , and a downward distribution of particles. We see that about $50 \%$ of the particles interact at densities greater than $10^{14} \mathrm{H} \mathrm{cm}^{-3}$.

Important information about the acceleration and transport of accelerated electrons and ions can be obtained by a comparison of the time histories observed in different energy bands of the hard X-ray and gamma-ray spectra. Chupp (1990) mentions a preliminary study of $S M M$ data indicating that the peak rate in an individual burst observed in the $4.1-6.4 \mathrm{MeV}$ energy band (typically due primarily to ion interactions) was delayed between $2 \mathrm{~s}$ and $45 \mathrm{~s}$ from the corresponding maximum observed in the hard X-ray continuum near $300 \mathrm{keV}$. This delay appears to be proportional to the rise time of the pulse. In Figure 4 we plot the time profiles of two flares observed by $S M M$ in three energy bands. We expect the low-energy band to be dominated by electron bremsstrahlung and the 4.1 - 6.4 MeV band to have a large contribution from nuclear gamma rays; the band near $300 \mathrm{keV}$ will have small contributions from instrumentally de- 

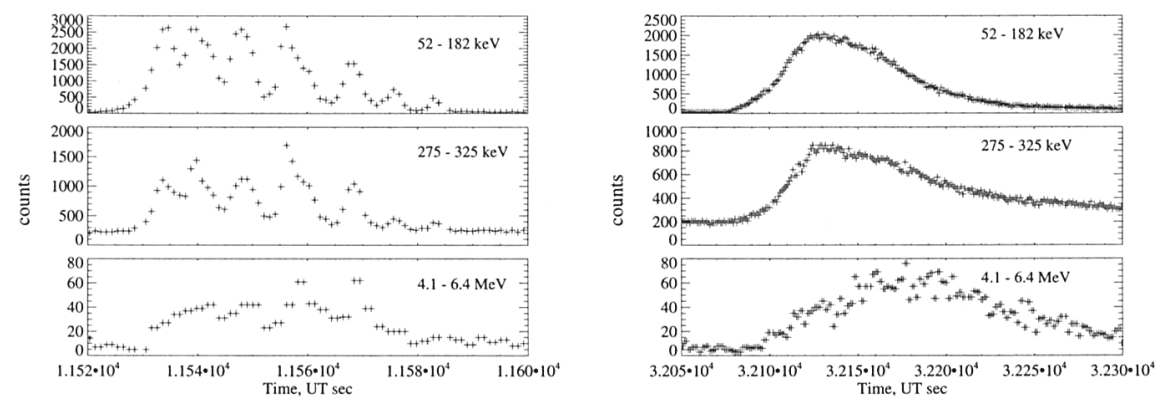

Figure 4. Comparison of flare time histories in three energy bands. Left panel, entire 1980 June 7 impulsive flare; right panel, one peak in the 1988 Dec. 16 flare.

graded nuclear radiation and the positronium continuum in addition to electron bremsstrahlung. The peaks in the low and high-energy bands in the June 7 flare appear to coincide; in contrast the Dec. 16 peak shows increasing delay with energy. In order to quantify this finding, we have performed a cross correlation analysis of the two higher energy bands with the low-energy band for each flare. We find $\sim 1.5 \mathrm{~s}$ and $\sim 35 \mathrm{~s}$ delays between the hard X-ray and $4.1-6.4 \mathrm{MeV}$ bands in the June 7 flare and Dec 16 flares, respectively. These disparate delays and the greatly different rise times for the two flares illuminate the findings discussed by Chupp (1990). Hulot et al. (1992) discuss this delay in terms of a trap-plus-precipitation model. We are currently performing this analysis on a large sample of flares in order to determine whether these delays are due to different acceleration processes for electrons and ions, or to transport effects.

\section{Spectroscopy of 2002 July 23 Solar Flare}

In this section we discuss RHESSI spectroscopic measurements of the 2002 July 23 solar flare in the context of our understanding of particle acceleration, transport, and interaction in a flaring solar atmosphere. Lin et al. (2003) summarize the characteristics of the flare and the highlights of the RHESSI observations (see also other papers in the same issue of ApJ). The X4.8 class flare erupted

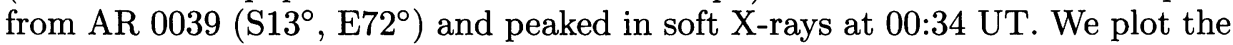
background-corrected spectrum observed by RHESSI from 00:27:20 to 00:43:20 UT in Figure 5. It has been fit by folding an incident photon spectrum containing electron bremsstrahlung and nuclear lines through the instrument response function. The overall fit to the data is good and the individual components of the fit are shown separately in the figure; Share et al. (2003a) list the best fitting parameters. The curves show the full instrumental response to the incident radiation, including both events in which the full energy has been absorbed and those in which only part of the energy has been absorbed (as reflected in the lower energy Compton continuum). The electron bremsstrahlung is best fit by a broken power law (indices 2.9 and 2.1 below and above $\sim 550 \mathrm{keV}$ ). Holman et al. (2003) discuss the low-energy portion of this spectrum in detail. The hardening observed in the spectrum cannot be fully explained by relativistic rise in the 


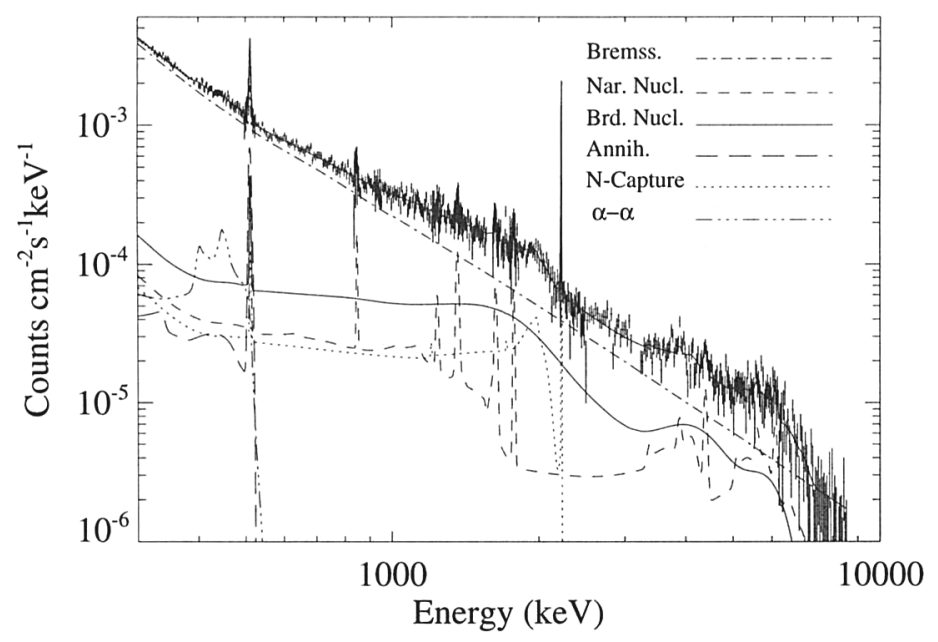

Figure 5. Count spectrum of the 2002 July 23 solar flare with fits revealing different components.

bremsstrahlung production cross section (see earlier discussion) and therefore must be due to an additional hard component in the electron spectrum. There is preliminary evidence that the harder portion of the electron spectrum may be delayed in time relative to the lower energy section, consistent with this suggestion (this study was performed at $20 \mathrm{~s}$ resolution and needs to be confirmed using higher time resolution data).

\subsection{Nuclear De-excitation Lines}

Our fit to the nuclear de-excitation lines plotted in Figure 5 is separated into two overall components. The first comprises de-excitation lines formed in interactions of protons and alpha particles with the ambient solar material (dashed curve). We call these features the 'narrow' nuclear component. For $S M M$ flares Share et al. (2002) have shown that these lines are redshifted from their laboratory energies for flares at small heliocentric angles but not at heliocentric angles near $73^{\circ}$ and above. This is consistent with a downward isotropic particle distribution. Surprisingly, the RHESSI lines are redshifted, as can be seen by the fits made to six of these lines in Figure 6 (Smith et al. 2003). The best fits to the lines are shown by the heavy solid curves while the thin solid curves show the expected lines without any shift. Such redshifts are not expected for a downward isotropic distribution of particles at heliocentric angles near $73^{\circ}$. Shown plotted in Figure 7 are the percentage redshifts of the RHESSI lines. There is a clear correlation with inverse of the atomic mass, as one would expect for nuclear recoil. The $S M M$ observations at this angle are shown for comparison. Smith et al. (2003) offer two explanations for the shifts observed by RHESSI: 1) the magnetic loops containing the particles are tilted toward Earth or 2) the spectrum of particles is very hard and they are strongly beamed.

There are two other de-excitation lines that are sensitive to the directionality of the interacting particles. These are the ${ }^{7} \mathrm{Be}(429 \mathrm{keV})$ and ${ }^{7} \mathrm{Li}(478 \mathrm{keV})$ 

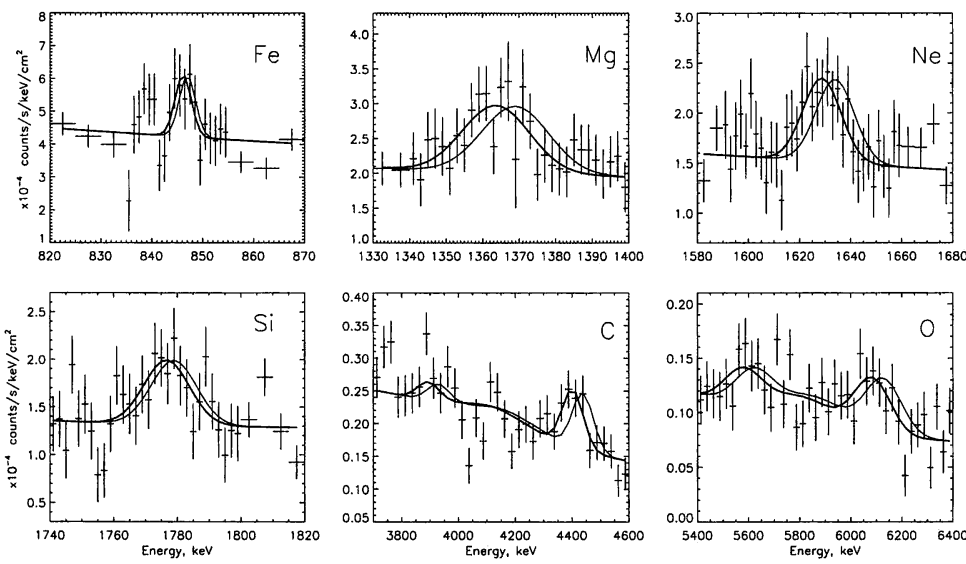

Figure 6. Fits to individual lines in the RHESSI spectrum. Thick curve is best fit; thin curve is fit to an unshifted line. Reprinted courtesy of the Astrophysical Journal (c) 2003).

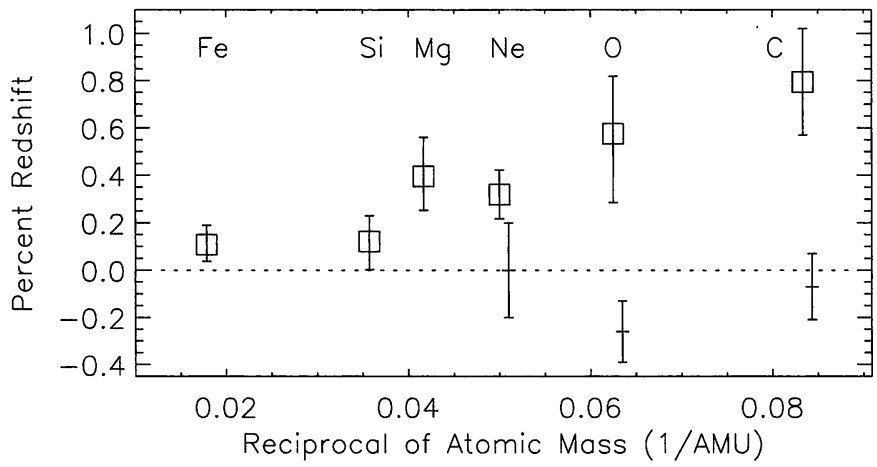

Figure 7. Redshift of de-excitation lines as function of inverse atomic mass. Squares are RHESSI data, points with dashes are from the average of 5 flares observed by $S M M$ at $\sim 74^{\circ}$ heliocentric angle. Reprinted courtesy of the Astrophysical Journal (c) 2003) 


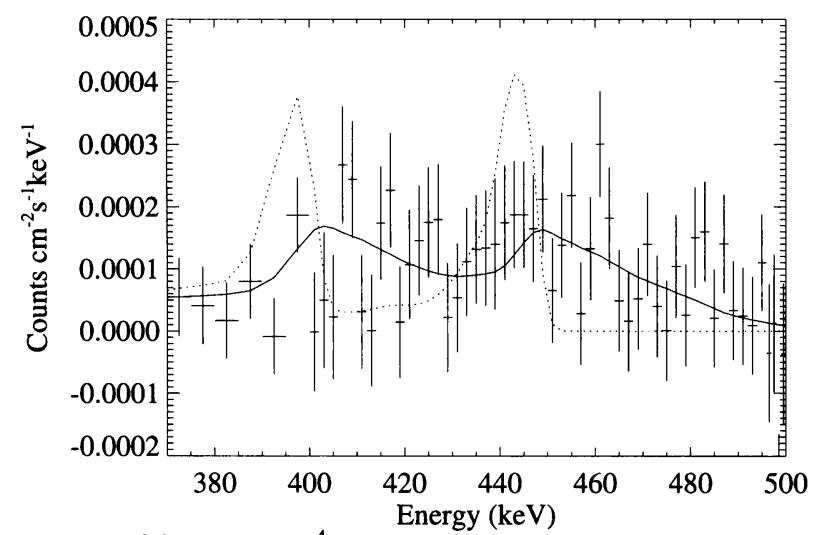

Figure 8. RHESSI solar $\alpha-{ }^{4} \mathrm{He}$ line spectrum. Solid curve is best fit for saturated pitch-angle scattering in the corona. Dotted curve is fit for a downward beam at 73 degrees heliocentric angle

lines formed in fusion of accelerated $\alpha$-particles with chromospheric ${ }^{4} \mathrm{He}(\mathrm{Ko}-$ zlovsky and Ramaty 1974). The expected line shapes for different angular distributions can be calculated using algorithms described by Ramaty, Kozlovsky, \& Lingenfelter (1979). We have compared these shapes with the backgroundsubtracted spectrum observed by RHESSI between 370 and $500 \mathrm{keV}$ plotted in Figure 8 (Share et al. 2003b). A particle distribution produced by strong pitchangle scattering in the corona provides the best fit to the data, but isotropic, downward-isotropic, and fan-beam distributions also provide acceptable fits. We can rule out a downward-beamed distribution (shown by the dotted lines in Figure 8) with high confidence. The data are consistent with a forward isotropic distribution of interacting particles confined in magnetic loops tilted toward Earth by $\sim 40^{\circ}$. Thus only Smith et al.'s (2003) first explanation for the redshifts observed in the other de-excitation lines is consistent with the $\alpha-{ }^{4} \mathrm{He}$ line shapes.

\subsection{Accelerated and Ambient Helium Abundances}

The intensity of the $\alpha-{ }^{4} \mathrm{He}$ lines in flares observed by the $S M M$ spectrometer has been found to be more intense than expected for assumed accelerated $\alpha / \mathrm{p}$ and ambient ${ }^{4} \mathrm{He} / \mathrm{H}$ abundances of $\sim 10 \%$ (Share \& Murphy 1997). Kozlovsky, Murphy, \& Share (2004) set a $1 \sigma$ lower limit of $\sim 0.35$ to the $\alpha / p$ ratio from the 2003 July 23 flare, consistent with the $S M M$ observations. Mandzhavidze, Ramaty, \& Kozlovsky (1997) suggested a method to determine whether the high line fluxes are due to an elevated $\alpha / \mathrm{p}$ ratio and/or to an elevated ambient ${ }^{4} \mathrm{He} / \mathrm{H}$ ratio. This requires comparison of the fluxes in lines produced by $\alpha-{ }^{56} \mathrm{Fe}$ $(339 \mathrm{keV})$ and $\mathrm{p}^{56} \mathrm{Fe}(847 \mathrm{keV})$ lines. There is evidence for a weak line at 339 $\mathrm{keV}$ in data obtained with moderate resolution $\mathrm{NaI}$ spectrometers, consistent with an elevated $\alpha / \mathrm{p}$ ratio (Share \& Murphy 1998). Such a study using the high-resolution Ge detector on RHESSI is planned.

The photospheric abundance of ${ }^{3} \mathrm{He}$ is estimated to be only about $\sim 2 \times 10^{-5}$ of $\mathrm{H}$ based primarily based on solar wind, coronal, and meteoritic measure- 


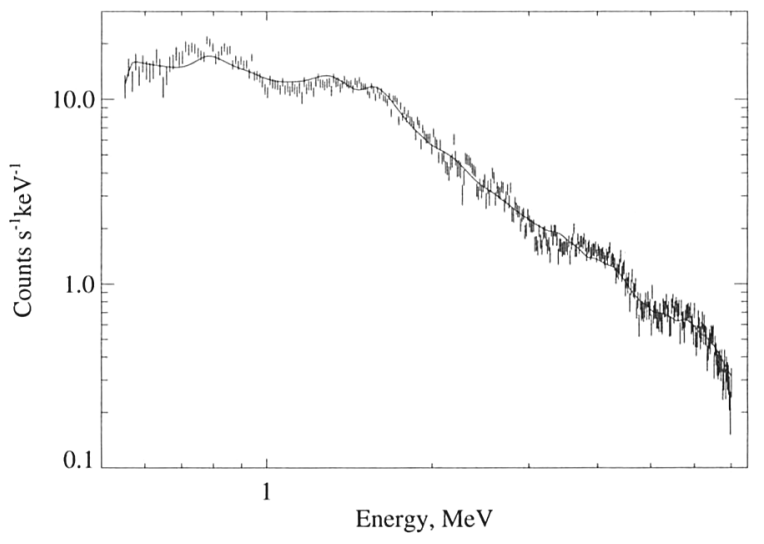

Figure 9. Broad nuclear line component observed in the summed spectrum of 19 flares observed by $S M M$. The curve shows the calculated shape for accelerated particles with impulsive composition, $\mathrm{a} / \mathrm{p}=$ 0.5 , power-law index 4.5 , and downward isotropic distribution. The unresolved contribution is from Ramaty, Kozlovsky, \& Lingenfelter 1979.

ments. In principle it can also be determined from the time profile of the narrow $2223 \mathrm{keV}$ neutron-capture line (Wang \& Ramaty, 1994) formed after flare-produced neutrons slow down and are captured on ${ }^{1} \mathrm{H}$. This is true because the ${ }^{3} \mathrm{He}(n, p)^{3} \mathrm{H}$ reaction has a large cross section and produces no radiation; it therefore shortens the time delay of the capture line. Murphy et al. $(2003 ; 2004)$ discuss observations of the $2223 \mathrm{keV}$ time profile measured by RHESSI and review previous observations relating to the determination of the ${ }^{3} \mathrm{He}$ abundance. They point out that various parameters contribute to the observed profile in addition to the concentration of ${ }^{3} \mathrm{He}$. These include: 1 . the spectrum of the accelerated particles, 2 . the angular distribution of the accelerated particles and their pitch-angle scattering in the corona, 3 . the tilt of the magnetic loops constraining the particles relative to the solar surface, and 4 . the density model of the solar atmosphere during the flare. Because of uncertainties introduced be these additional parameters Murphy et al. (2004) conclude that current gammaray data are insufficient to reliably estimate the photospheric ${ }^{3} \mathrm{He}$ abundance.

${ }^{3} \mathrm{He}$ is strikingly enhanced in impulsive solar energetic particle events, reaching ${ }^{3} \mathrm{He} /{ }^{4} \mathrm{He}$ ratios $\geq 1$, in marked contrast to the photospheric abundance. This excess has been explained by resonant acceleration by electromagnetic waves (see Paesold, Kallenbach, \& Benz 2003, and references within). There is also evidence for enhanced ${ }^{3} \mathrm{He}$ in accelerated particles impacting the Sun from gamma-ray observations. The ${ }^{16} \mathrm{O}\left({ }^{3} \mathrm{He}, p\right){ }^{18} \mathrm{~F}^{*}$ reaction produces a gamma-ray line at $937 \mathrm{keV}$ (Mandzhavidze et al. 1997). Studies with SMM using this line suggest that the ${ }^{3} \mathrm{He} /{ }^{4} \mathrm{He}$ ratio could be $\sim 0.1$ or higher (Share \& Murphy 1998; Mandzhavidze, Ramaty, \& Kozlovsky 1999). Kozlovsky, Murphy, \& Share (2004) set a $99 \%$ upper limit of 0.23 on the ${ }^{3} \mathrm{He} /{ }^{4} \mathrm{He}$ ratio using the high-resolution RHESSI observation of the 2002 July 23 flare. 

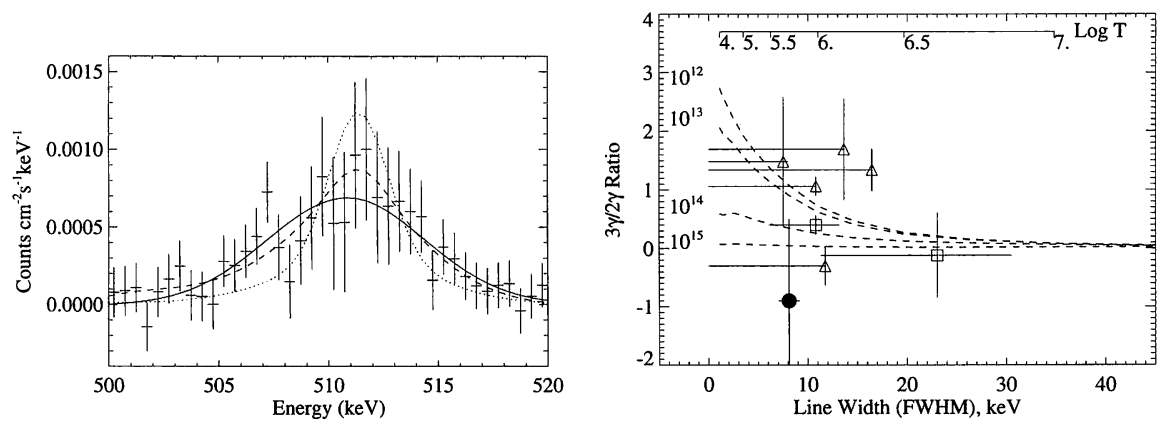

Figure 10. Left panel: spectrum of solar $511 \mathrm{keV}$ line with Gaussian fit (solid), and fits from calculated line shapes at $5000 \mathrm{~K}$ (dotted) and $6000 \mathrm{~K}$ (dashed) in a quiet solar atmosphere. Right panel: Comparison of RHESSI (filled circle) and SMM measurements of the $3 \gamma / 2 \gamma$ ratio vs $511 \mathrm{keV}$ line width and temperature for a fully ionized medium. Curves show calculations for different densities. Reprinted courtesy of the Astrophysical Journal (c) 2003)

\subsection{Accelerated Heavy Ions}

From the fit to the overall RHESSI spectrum shown in Figure 5 we note that the broad nuclear lines (solid curve) can contribute up to about $25 \%$ of the total flux in certain energy bands (e.g. near the ${ }^{12} \mathrm{C}$ line at $4.4 \mathrm{MeV}$ ). Share \& Murphy (1999) presented the broad line spectrum from $S M M$; it is shown in Figure 9, after narrow lines and bremsstrahlung have been subtracted from the flare spectrum. Highly Doppler broadened lines from accelerated ${ }^{56} \mathrm{Fe},{ }^{12} \mathrm{C}$, and ${ }^{16} \mathrm{O}$ appear to be resolved. The shape of the spectrum is in reasonable agreement with calculations; the major uncertainty is the shape of the unresolved nuclear continuum that can dominate the spectrum in the 1 to $2 \mathrm{MeV}$ range.

\subsection{Positron Production and Annihilation}

RHESSI made the first high-resolution observation of the solar positron-electron annihilation line during the July 23 flare (Share et al. 2003a). We plot the measured line in the left panel of Figure 10, after removing contributions from the instrument and atmosphere. It is significantly broader than either of these backgrounds. The observed width of the line is consistent with annihilation at temperatures of either $\sim 6 \times 10^{3} \mathrm{~K}$ or $4-7 \times 10^{5} \mathrm{~K}$. There appear to be difficulties with an origin at either location, however.

Calculations indicate that there is only a narrow range of temperatures around $6 \times 10^{3} \mathrm{~K}$, and only in a quiet solar atmosphere, where the line shape is dominated by the formation of positronium in flight (the positron replaces the proton in the hydrogen atom). The positronium can be formed in either the singlet or triplet state (Crannell et al. 1976). When it annihilates from the singlet state, it emits two $511 \mathrm{keV} \gamma$ rays $(2 \gamma)$ in the center-of-mass frame; the lines are broadened by the velocity of the positronium. The dashed line in the left panel of Figure 10 shows that the line produced at $6 \times 10^{3} \mathrm{~K}$ provides a good fit to the data; however, the line produced at $5 \times 10^{3} \mathrm{~K}$ is too narrow 
(dotted line). When positronium annihilates from the triplet state, it emits three photons each with energy $<511 \mathrm{keV}$ and forms a continuum ( $3 \gamma$ continuum). At this location in the solar atmosphere, one expects a $3 \gamma / 2 \gamma$ ratio $>2.7$. The RHESSI upper limit on the $3 \gamma$ continuum yields a ratio that is only consistent with this requirement with $<4 \%$ confidence.

The width of the annihilation line is also consistent (solid curve in the left panel of Figure 10) with thermal broadening (Gaussian width of $8.1 \pm 1.1 \mathrm{keV}$ ) in a plasma at $4-7 \times 10^{5} \mathrm{~K}$. In a quiet solar atmosphere, these temperatures are only reached in the transition region at densities $\leq 10^{12} \mathrm{H} \mathrm{cm}^{-3}$. Although, the $S M M$ spectrometer could not resolve the annihilation line, it made observations of the $3 \gamma / 2 \gamma$ ratio in seven flares. We plot the measured $3 \gamma / 2 \gamma$ ratio vs the measured line widths (and temperatures) for the SMM and RHESSI flares in the right panel of Figure 10; the dashed curves show the calculated relationships for densities from $10^{12}-10^{15} \mathrm{H} \mathrm{cm}^{-3}$ in an ionized atmosphere. The RHESSI and all but two of the $S M M$ measurements are consistent with densities $\leq 10^{12} \mathrm{H} \mathrm{cm}^{-3}$. We see from Figure 3 that $<10 \%$ of the $\mathrm{p}$ and $\alpha$ interactions producing positrons occur at these low densities. The question is how can the positrons annihilate at such low densities. Kozlovsky et al. 2004 show that positrons produced by ${ }^{3} \mathrm{He}$ interactions form higher in the solar atmosphere; however, in order to explain the line width, it would require a much higher ${ }^{3} \mathrm{He} /{ }^{4} \mathrm{He}$ ratio than the upper limit set for this flare by RHESSI. Alternatively, all the observations are consistent with densities $>10^{12} \mathrm{H} \mathrm{cm}^{-3}$. But such densities require formation of a substantial mass of atmosphere at transition region temperatures.

This work was supported by NASA DPR's W19746 and W10049 and by the Office of Naval Research.

\section{References}

Avrett, C. W. 1981, in The Physics of Sunspot, ed. L.E. Cram \& J. H. Thomas (Sunspot: Sacramento Peak Observatory), 235

Aschwanden, M. J. 2002, Space Sci.Rev., 101, 1

Aschwanden, M. J., et al. 1996, ApJ, 470, 1198

Chupp, E.L. 1990, Science, 250, 229

Chupp, E.L., et al. 1973, Nature, 241, 333

Chupp, E.L., et al. 1987, ApJ, 318, 913

Crannell, C. J., Joyce, G., Ramaty, R., \& Werntz, C. 1976, ApJ, 210, 582

Hua, X.-M., et al. 2002, ApJS, 140, 563

Hua, X.-M., Ramaty, R., \& Lingenfelter, R. E. 1989, ApJ, 341, 516

Hulot, E., et al. 1992, A\&A, 256, 273

Kanbach, G., et al. 1993, A\&AS, 97, 349

Kozlovsky, B., Murphy, R. J., \& Share, G. H. 2004, submitted for publication in ApJ

Kozlovsky, B. \& Ramaty, R. 1974, ApJ, 191, L43

Kozlovsky, B., Murphy, R. J., \& Ramaty, R. 2002, ApJS, 141, 523

Lin, R. P., et al. 2003, ApJ, 595, L69 
Mandzhavidze, N., Ramaty, R., \& Kozlovsky, B. 1997, ApJ, 489, L99

Masuda, S., et al. 1994, Nature 371, 495

Mctiernan, J. M., \& Petrosian, V. 1990, ApJ359, 541

Miller, J. A., 2000, in ASP Conf. Ser. Vol. 206, High Energy Solar PhysicsAnticipating HESSI, ed. R. Ramaty \& N. Mandzhavidze, (San Francisco: ASP), 145

Miller, J. A., \& Ramaty, R. 1989, ApJ, 344, 973

Miller, J. A., et al. 1997, J. Geophys. Res. 102: (A7), 14631

Murphy, R. J., et al. 2003, ApJ, 595, L93

Murphy, R. J., et al. 2004, in preparation

Paesold, G., Kallenbach, R., \& Benz, A. O. 2003, ApJ, 582, 495

Ramaty, R., Kozlovsky, B., \& Lingenfelter, R. E. 1979, ApJS, 40, 487

Ramaty, R., Mandzhavidze, N., Kozlovsky, B., \& Murphy, R. J. 1996, ApJ, 445, $\mathrm{L} 193$

Share, G. H., \& Murphy, R. J. 1995, ApJ, 452, 933

Share, G. H., \& Murphy, R. J. 1997, ApJ, 485, 409

Share, G. H., \& Murphy, R. J. 1998, ApJ, 508, 876

Share, G. H., \& Murphy, R. J. 1999, Proc. $26^{\text {th }}$ Int. Cos. Ray Conf., 6, 13

Share, G.H., et al. 2002, ApJ, 573, 464

Share, G. H., et al. 2003a, ApJ, 595, L85

Share, G. H., et al. 2003b, ApJ, 595, L89

Smith, D. H., et al. 2003, ApJ, 595, L81

Vestrand, W. T., et al. 1987, ApJ, 322, 1010

Wang, H.T. \& Ramaty, R. 1974, Sol. Phys., 36, 129 